\title{
Communication \\ Direct RNA Sequencing Reveals SARS-CoV-2 m6A Sites and Possible Differential DRACH Motif Methylation among Variants
}

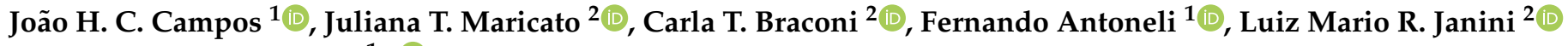 \\ and Marcelo R. S. Briones ${ }^{1, *(\mathbb{D})}$ \\ 1 Center for Medical Bioinformatics, Escola Paulista de Medicina, Federal University of São Paulo (UNIFESP), \\ São Paulo 04039032, Brazil; joao.heima@gmail.com (J.H.C.C.); fernando.antoneli@unifesp.br (F.A.) \\ 2 Department of Microbiology, Immunology and Parasitology, Escola Paulista de Medicina, \\ Federal University of São Paulo (UNIFESP), São Paulo 04023062, Brazil; juliana.maricato@unifesp.br (J.T.M.); \\ ctbsantos@unifesp.br (C.T.B.); janini@unifesp.br (L.M.R.J.) \\ * Correspondence: marcelo.briones@unifesp.br; Tel.: +55-11-5576-4537
}

\section{check for}

updates

Citation: Campos, J.H.C.; Maricato, J.T.; Braconi, C.T.; Antoneli, F.; Janini, L.M.R.; Briones, M.R.S. Direct RNA Sequencing Reveals SARS-CoV-2 m6A Sites and Possible Differential DRACH Motif Methylation among Variants. Viruses 2021, 13, 2108. https://doi.org/10.3390/v13112108

Academic Editor:

Kenneth Lundstrom

Received: 3 September 2021

Accepted: 13 October 2021

Published: 20 October 2021

Publisher's Note: MDPI stays neutral with regard to jurisdictional claims in published maps and institutional affiliations.

Copyright: (c) 2021 by the authors. Licensee MDPI, Basel, Switzerland. This article is an open access article distributed under the terms and conditions of the Creative Commons Attribution (CC BY) license (https:// creativecommons.org/licenses/by/ $4.0 /)$.

\begin{abstract}
The causative agent of COVID-19 pandemic, SARS-CoV-2, has a 29,903 bases positive-sense single-stranded RNA genome. RNAs exhibit about 150 modified bases that are essential for proper function. Among internal modified bases, the $N^{6}$-methyladenosine, or m6A, is the most frequent, and is implicated in SARS-CoV-2 immune response evasion. Although the SARS-CoV-2 genome is RNA, almost all genomes sequenced thus far are, in fact, reverse transcribed complementary DNAs. This process reduces the true complexity of these viral genomes because the incorporation of dNTPs hides RNA base modifications. Here, we present an initial exploration of Nanopore direct RNA sequencing to assess the m6A residues in the SARS-CoV-2 sequences of ORF3a, E, M, ORF6, ORF7a, ORF7b, ORF8, N, ORF10 and the 3'-untranslated region. We identified fifteen m6A methylated positions, of which, six are in ORF N. Additionally, because m6A is associated with the DRACH motif, we compared its distribution in major SARS-CoV-2 variants. Although DRACH is highly conserved among variants, we show that variants Beta and Eta have a fourth position $\mathrm{C}>\mathrm{U}$ change in DRACH at 28,884b that could affect methylation. This is the first report of direct RNA sequencing of a Brazilian SARS-CoV-2 sample coupled with the identification of modified bases.
\end{abstract}

Keywords: SARS-CoV-2; COVID-19; m6A; direct RNA sequencing; RNA methylation; Epitranscriptomics

\section{Introduction}

RNA viruses are causative agents of major human transmissible diseases such as influenza, poliomyelitis, measles and COVID-19 [1-5]. In the Baltimore classification, viruses with RNA genomes comprise groups III, IV, V and VI, while DNA viruses are in groups I, II and VII [6,7]. COVID-19 is a highly contagious viral disease with severe respiratory, inflammatory and thrombotic manifestations [8,9]. The COVID-19 pandemic is caused by a Beta coronavirus, SARS-CoV-2, included in Group IV of the Baltimore classification [10,11]. In SARS-CoV-2, the RNAs serve as information storage when packaged into the viral particle and as mRNAs for viral protein synthesis upon infection of mammalian cells [12,13].

The SARS-CoV-2 genome consists of a positive-sense single-stranded strand RNA with 29,903 bases [13]. There are approximately 150 different base modifications in all RNA species, and these modified bases are essential for proper translation, splicing and RNA metabolism [14]. Among these modified bases, the $N^{6}$-methyladenosine (m6A) is the most frequent internal base modification, and is found in viruses with exclusive cytoplasm replication, such as Zika Virus, Dengue virus and Hepatitis C virus [15,16]. The methylated base m6A is implicated in SARS-CoV-2's evasion of the host immune response 
because the methylated viral RNA does not interact with the host protein RIG-I (retinoic acid-inducible gene I) responsible for the type-1 interferon (IFN1) response, an activator of immune pathways $[14,17,18]$. The viral RNA is m6A methylated by the host's methylases METTL3, METTL14, WATAP and KIAA1429, called "writers", and demethylated by FTO and ALKBH5, called "erasers", which normally demethylate the host's RNAs [19]. Knockdown of METTL3 significantly reduces the SARS-CoV-2 methylation and blocks the viral mechanism of RIG-I binding inhibition [17].

Almost all the SARS-CoV-2 genomes sequenced thus far are reverse transcribed complementary DNAs (cDNAs), although the genome is, in fact, RNA [20]. Reverse transcription provides a fast, practical, PCR prone method for sequencing the SARS-CoV-2 genome. However, it reduces the true complexity of these viral genomes. The incorporation of dNTPs in the first strand cDNA chain makes the RNA base modifications, present in the RNA template chain, mostly indistinguishable from unmodified bases and sequencing errors [21]. RNA modified bases are critical for proper biological function and are involved in several diseases, encompassing the field of Epitranscriptomics Medicine [19]. Although different technologies have been used for the identification of modified bases in mRNAs and viral RNAs, they require substantial quantities of material that precludes single-cell analysis and low abundance samples. Additionally, the antibody-dependent methylation analysis does not provide nucleotide level accuracy of modified bases [14,17,18,22].

Oxford Nanopore Technology (ONT) has been used for SARS-CoV-2 whole-genome cDNA sequencing [23]. Additionally, this same technology has been used for direct RNA sequencing of SARS-CoV-2 [24-27]. The major advantage of ONT direct RNA sequencing over cDNA sequencing is the identification of modified bases. Two previous studies on SARS-CoV-2 direct RNA sequencing did not couple the genetic analysis with base modification identification, while a third study detected the $5 \mathrm{mC}$ methylation using this technology [24,26,27]. A fourth study detected sgRNAs in supernatants of Calu-3 cells (human lung epithelial) using direct RNA sequencing and detected modified bases but not specified by type [25].

In the present study, we assessed the potential of the Nanopore direct RNA sequencing for the identification of m6A residues, at nucleotide level resolution, in the SARS-CoV-2 genome. For this, we analyzed direct RNA sequencing reads of open reading frames (ORFs) $3 \mathrm{a}, \mathrm{E}, \mathrm{M}, 6,7 \mathrm{a}, 7 \mathrm{~b}, 8, \mathrm{~N}, 10$ and the $3^{\prime}$-untranslated region [22]. In addition, since $\mathrm{m} 6 \mathrm{~A}$ is associated with the DRACH motif $(D=G / A / U, R=G / A, H=A / U / C)$, we compared the DRACH distribution in major SARS-CoV-2 variants to verify if potential variant-specific alterations in m6A methylation patterns occur in SARS-CoV-2 evolution [28].

\section{Materials and Methods}

\subsection{Cell Cultures and SARS-CoV-2 Infection}

All procedures for viral isolation and initial passages were performed in a biosafety level 3 laboratory (BLS3), in accordance with WHO recommendations and under the laboratory biosafety guidance required for SARS-CoV-2 at the BLS3 facilities at the Federal University of São Paulo. SARS-CoV-2 stock was kindly provided by Prof. José Luiz Proença-Módena (University of Campinas-UNICAMP, SP, Brazil).

For SARS-CoV-2 infection, the Vero E6 cell line $\left(\mathrm{ATCC}^{\circledR}{ }^{\circledR}\right.$ CRL-1586 ${ }^{\mathrm{TM}}$ ) was maintained in Minimum Essential Medium (MEM; Gibco) supplemented with $10 \%$ fetal bovine serum (FBS) (Gibco) and 1\% penicillin/streptomycin (Gibco). Vero E6 cells were kept in a humidified $37{ }^{\circ} \mathrm{C}$ incubator with $5 \% \mathrm{CO}_{2}$. After reaching an $80 \%$ confluent monolayer, cells were seeded in 24-well plates at a density of $5 \times 10^{5}$ cells per well. Cells were infected at $1 \times 10^{5} \mathrm{PFU} /$ well (MOI 0.2) with SARS-CoV-2 lineage B [29], 3rd passage and kept for $2 \mathrm{~h}$ at $37^{\circ} \mathrm{C}$ with $5 \% \mathrm{CO}_{2}$ in MEM supplemented with $2.5 \% \mathrm{FBS}$ and $1 \%$ penicillin/streptomycin. Cells were rinsed with $1 \times$ PBS to remove attached viral particles, and fresh MEM with $10 \%$ FBS was added to the cultures. After $48 \mathrm{~h}$, the cell cultures were halted and used for supernatant harvesting [30]. 


\subsection{RNA Isolation}

RNA samples from culture supernatants were extracted using viral QIAamp Viral RNA Mini Kit (Qiagen, Germantown, MD, USA). Briefly, $350 \mu \mathrm{L}$ of supernatants were centrifuged at $3000 \mathrm{rpm}$ for $5 \mathrm{~min}$ to remove cell debris and transferred to new tubes containing $550 \mu \mathrm{L}$ of lysis buffer (AVL—provided with the kit) and RNA isolation was performed according to the manufacturer's instructions. RNA samples were quantitated with Nanodrop (Thermo Fischer Scientific, Waltham, MA, USA).

\subsection{Direct RNA Sequencing}

For RNA sequencing, $9.5 \mu \mathrm{L}$ of RNA containing $\approx 50 \mathrm{ng}$ of RNA, from Vero E6 cells supernatant, were used for construction of RNA sequencing libraries with the Nanopore RNA Sequencing kit SQK-RNA002 following the manufacturer's protocol with cDNA synthesis (Oxford Nanopore Technologies, Oxford Science Park, Oxford, UK). The RNA libraries were loaded and run on a MinION device (Oxford Nanopore Technologies) with flowcell FLO-MIN106 for $40 \mathrm{~h}$ and 1.59 million reads were generated. Raw data (fast5 files) were used for basecalling with Guppy (v-5.0.11) in high-accuracy mode.

\subsection{Assembly}

The resulting fastq reads were aligned to the SARS-CoV-2 reference (GISAID ID: EPI_ISL_413016) with minimap2 (v-2.21-r1071) [31]. The resulting sam files were converted to bam files and all reads were sorted and indexed according to the reference coordinates using samtools (v-1.13) [32]. The "index" and "eventalign" modules of nanopolish (v-0.13.3) were used to generate an index of base call reads for the signals measured using the sequencer, and to align events to the reference transcriptome, checking for differences in current that may suggest modifications in the base.

\subsection{Methylation Analysis}

The probability of methylation in DRACH motifs was calculated with m6anet (v-0.1.1-pre) [33], as recommended, (I) by preprocessing the segmented raw signal file with "m6anet-dataprep", and (II) running m6anet over data using "m6anet-run_inference".

\subsection{DRACH Motif Comparison}

Comparative analysis and annotation of DRACH motifs [28], identified with m6Anet (v-01.1-pre) [34], among SARS-CoV-2 variants were carried out using Geneious v-10.4 (http:/ / www.geneious.com, accessed on 21 August 2021). Five sequences of each variant isolated and sequenced in Brazil were aligned using the Geneious aligner. For variant Eta only, one sequence from Brazil is available from GISAID (http:/ /www.gisaid.org, accessed on 21 August 2021) that is complete with high coverage and, therefore, samples from the US, France, Spain and Canada were used. The SARS-CoV-2 variants sequences analyzed are deposited in the GISAID database (http://www.gisaid.org, accessed on 21 August 2021) with accession numbers for Alpha (EPI_ISL_1133259, 1133268, 1133267, 1495029, 3316204), Beta (1966629, 1742275, 1966124, 1445171, 1716877), Gamma (3539883, 3539773, 3545813, 3545803, 3540000), Delta (3540039, 3540020, 3540001, 3460250, 3505224), Eta $(1583653,3502618,3535614,3490821,3493947)$, Lambda $(1445272,3010903,2928137$, $1966094,2617911)$ and Zeta $(3434818,2841610,3506974,3190295,1494963)$. 


\section{Results \\ 3.1. Direct RNA Sequencing and Assembly}

The assembly of the $3^{\prime}$-half of the SARS-CoV-2 RNA genome was obtained by mapping the RNA reads to GISAID ID: EPI_ISL_413016, the strain used for the infection of the Vero E6 cells (Figure 1A). The coverage varied from $30 \times$ to $1600 \times$ from $5^{\prime}$ to $3^{\prime}$ starting at ORF3a. As the RNA sequencing adapter is ligated to the $3^{\prime}$-ends of RNAs, the coverage is higher as it gets closer to the $3^{\prime}$-end. Several reads reached the "Spike" ORF but were not used for further analysis due to low coverage. The sequencing runs of $40 \mathrm{~h}$ were sufficient to obtain around 2000 reads corresponding to the $3^{\prime}$-half of the SARS-CoV-2 genome. The average sequence length was 787 bases and the mode 1350 bases. The global identity to the reference was $90 \%$. The phred scores of the assembled bases are between 20 and 30 . ORF N has a substantial coverage because of its proximity to the $3^{\prime}$-end $(\approx 1000 \times)$.

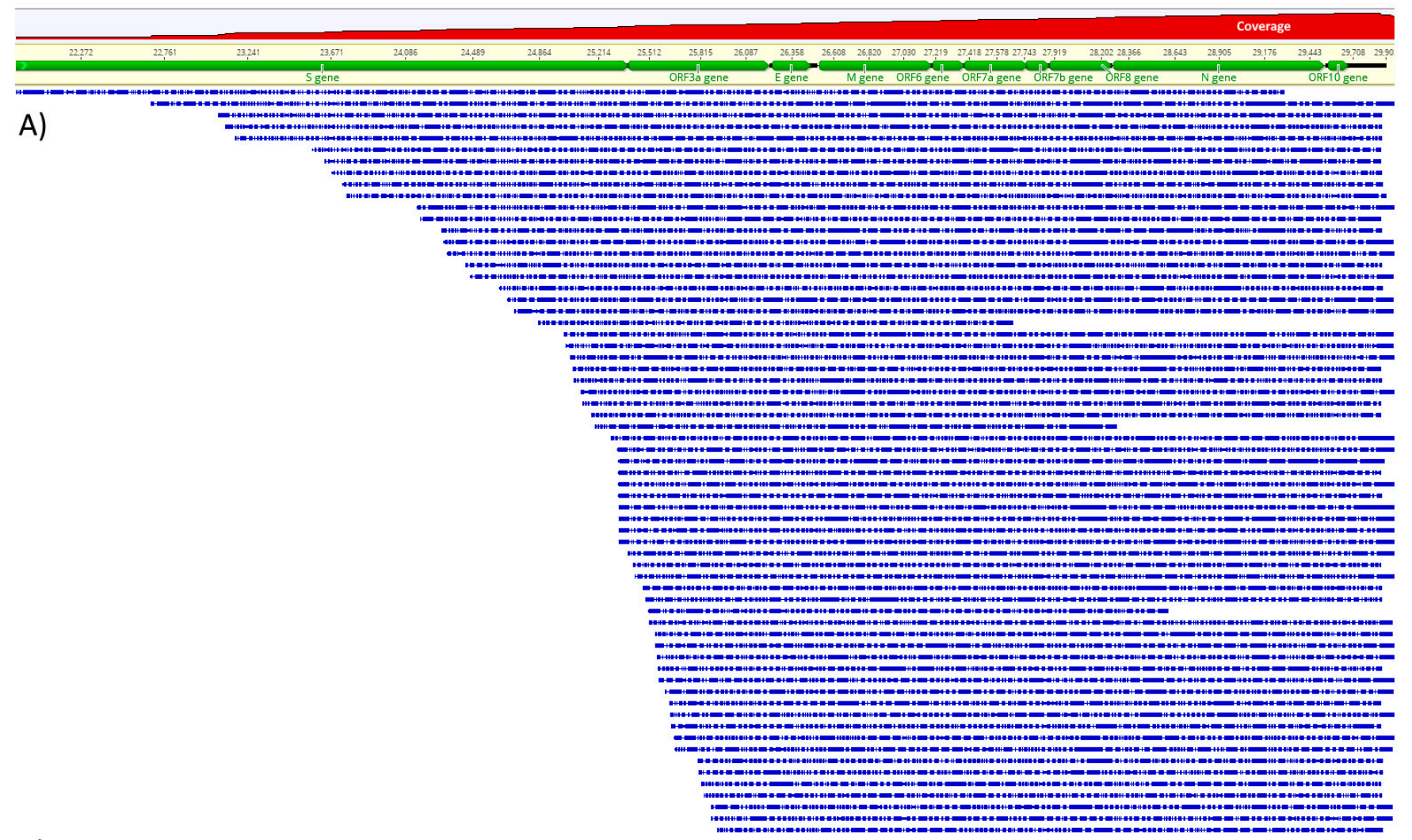

B)

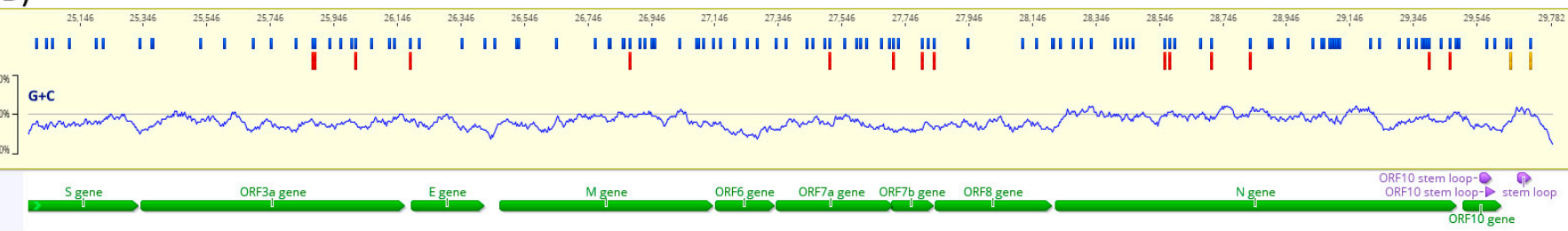

Figure 1. Assembly of Nanopore direct RNA reads to the reference sequence (A) and map of m6A methylation probability along the SARS-CoV-2 RNA (B). In (A), green horizontal bars indicate the ORFs, blue horizontal bars of decreasing size indicate the Nanopore reads and the red area at the top indicates the log scale coverage from $1 \times$ to $1600 \times$. In $(B)$, blue vertical bars indicate the DRACH motifs, red vertical bars indicate m6A ( $>50 \%$ probability) and the yellow vertical bars indicate two potential m6A with probabilities 0.38 and 0.44 in the $3^{\prime}$-untranslated region. The $\mathrm{G}+\mathrm{C}$ content is indicated in the plot just above the annotation. 


\subsection{Detection of $m 6 A$}

Direct RNA sequencing reads were used for the detection of m6A using the m6Anet tool, validated by systematic benchmark [35]. This method uses the reference sequence, the basecalled fastq files and the raw fast 5 files to identify the DRACH motifs and the raw signal data in fast 5 files with corresponding signal alterations associated with $\mathrm{m} 6 \mathrm{~A}$ to calculate the probability of bona fide methylation [35]. Using this approach, we identified 15 positions within DRACH with $\geq 50 \%$ methylation probability in at least one replicate (Figure 1B). Among these positions, 11 have more than $100 \times$ coverage and four positions have $>80 \%$ methylation probability (Table 1 ). The nucleocapsid region $(\mathrm{N})$ contains six putative $\mathrm{m} 6 \mathrm{~A}$ residues and at least $30 \mathrm{DRACH}$ motifs.

Table 1. Distribution and sequencing coverage of potential methylated adenosines in SARS-CoV-2 RNA genome. Coverage $>100 \times$ is underlined and probability $>80$ is in boldface as calculated by m6Anet [34]. After m6Anet analysis, only sites with coverage above 60 were considered. Position numbering according to Wuhan reference sequence (GenBank NC_045512). In Coverage and Probability columns, the results for two technical replicates are shown. The first numbers on these columns represent the experiment shown in Figure 1.

\begin{tabular}{ccccc}
\hline$\#$ & RNA Id-ORF & Position & Coverage & Probability \\
\hline 1 & EPI_ISL_413016-3a & 25,935 & $65 / 75$ & $0.5472 / 0.2749$ \\
2 & EPI_ISL_413016-3a & 25,940 & $67 / 61$ & $0.5094 / 0.5865$ \\
3 & EPI_ISL_413016-3a & 26,070 & $72 / 83$ & $0.6659 / 0.2497$ \\
4 & EPI_ISL_413016-3a/E & 26,241 & $83 / 100$ & $0.5480 / 0.3461$ \\
5 & EPI_ISL_413016-M & 26,933 & $162 / 295$ & $0.5102 / \mathbf{0 . 8 2 0 4}$ \\
6 & EPI_ISL_413016-7a & 27,562 & $266 / 679$ & $\mathbf{0 . 8 5 1 6} / 0.3973$ \\
7 & EPI_ISL_413016-7b & 27,764 & $309 / 849$ & $\mathbf{0 . 8 4 3 3} / \mathbf{0 . 8 9 4 6}$ \\
8 & EPI_ISL_413016-7b & 27,854 & $318 / 839$ & $\mathbf{0 . 8 7 6 1 / 0 . 8 7 0 9}$ \\
9 & EPI_ISL_413016-7b/8 & 27,892 & $357 / 859$ & $0.5770 / 0.4420$ \\
10 & EPI_ISL_413016-N & 28,616 & $699 / 812$ & $\mathbf{0 . 8 1 8 4} / 0.4929$ \\
11 & EPI_ISL_413016-N & 28,633 & $314 / 364$ & $0.5022 / 0.4134$ \\
12 & EPI_ISL_413016-N & 28,766 & $794 / 817$ & $0.5403 / 0.4098$ \\
13 & EPI_ISL_413016-N & 28,886 & $871 / 835$ & $0.7020 / 0.2212$ \\
14 & EPI_ISL_413016-N & 29,450 & $922 / 803$ & $0.5523 / 0.2701$ \\
15 & EPI_ISL_413016-N & 29,517 & $887 / 783$ & $0.5705 / 0.3598$ \\
\hline
\end{tabular}

\subsection{DRACH Motif Analysis}

As the DRACH motif is associated with m6A, we tested if the major SARS-CoV-2 variants, Alpha, Beta, Gamma, Delta, Eta, Lambda and Zeta, isolated in Brazil exhibited mutations within DRACH that differ between the variants. The alignment consisted of the Wuhan reference sequence (GenBank NC_045512), the Brazilian reference (GISAID ID: EPI_ISL_413016) and five sequences of major variants (Material and Methods). DRACH is highly conserved among variants; however, differences can be observed (Figure 2). Five sequences of the variant Beta and four of the variant Eta have a $\mathrm{C}>\mathrm{U}$ change in the fourth position of DRACH (at position 28,886) that could block methylation at this site (Figure 2A, D1). The methylation probability at this site is $70 \%$ and the coverage $871 \times$. Another change in DRACH that could probably interfere with methylation is a $\mathrm{C}>\mathrm{U}$ at 28,947 (Figure 2A, D2) in a single variant Zeta sequence, although the methylation probability at this site is $<50 \%$. Other DRACH variants observed are probably "silent", such as the four nucleotides insertion in the intergenic region between ORF8 and ORF N in the five sequences of variant Gamma (Figure 2B, D3). The insertion does not change the DRACH sequence. 


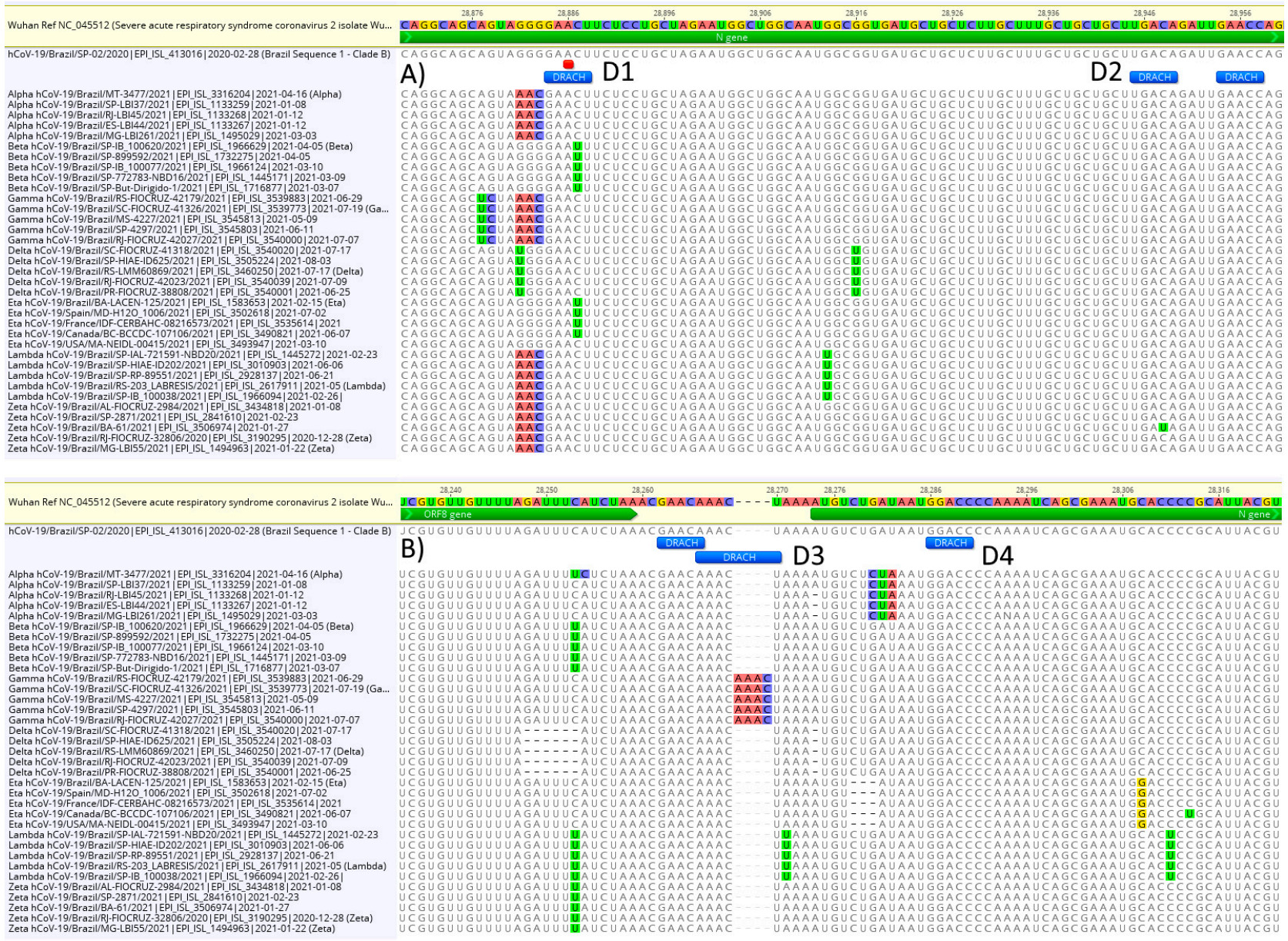

Figure 2. Variability of DRACH sequences among SARS-CoV-2 variants. In (A), the $\mathrm{C}>\mathrm{U}$ change at 28,886 disrupts the m6A (marked red) DRACH motif (D1) in five variant Beta sequences and four variant Eta sequences, while a single variant Zeta has a $\mathrm{C}>\mathrm{U}$ mutation that disrupts the DRACH motif (D2). In (B), a four bases insertion in the intergenic region ORF8/N does not disrupt DRACH (D3), while (D4) shows a fully conserved DRACH.

\section{Discussion}

RNA modification, or epigenomics, is a key factor in viral infections [14]. The methylation of RNA bases, either as the host's multitargets or viral RNAs, is involved in immunity and associated disease progression $[17,36]$. Various types of RNA methylation are involved in COVID-19 immunity and novel proposed treatments involve methylase inhibitors $[37,38]$. The Nanopore direct RNA sequencing offers a unique opportunity for the study of viral epigenomics [22,24]. Direct RNA sequencing is PCR-free, is not sequencing-by-synthesis and, therefore, not affected by PCR bias and synthesis errors. The modified bases are preserved and assessed directly with no need for chemical treatments or antibodies. Direct RNA sequencing of SARS-CoV-2 has been validated using orthogonal methods [27].

In the present study, we show that direct RNA sequencing of SARS-CoV-2 is a valuable tool for the assessment of the full complexity of this viral RNA genome. The identification of m6A was performed in supernatants of SARS-CoV-2-infected Vero E6 cells and, therefore, is enriched in genomic RNAs and not subgenomic RNA [25]. Sequencing was performed without PCR amplification or any other in vitro synthesis (Figure 1).

The mapping of m6A using direct RNA sequencing data was achieved by a comparison of raw and basecalled reads. The m6A pattern identified is consistent with the nucleocapsid region m6A enrichment observed using liquid chromatography-tandem mass spectrometry and methylated RNA immunoprecipitation sequencing (MeRIP-seq) [17]. The probability of $\mathrm{m} 6 \mathrm{~A}$, as calculated using the $\mathrm{m} 6 \mathrm{Anet}$ method, was $>50 \%$ and a minimum coverage of $60 \times$ in four positions and the recommended $100 \times$ coverage in 11 positions 
in the first experiment [35]. In Figure $1 \mathrm{~B}$, the m6A positions are confirmed by at least one experiment for $\mathrm{a} \geq 50 \%$ probability. Positions that are not methylated almost always have probabilities below $5 \%$. Our results suggest that the nucleocapsid region has more methylated sites (Table 1), which is consistent with previous studies using Met-RIP, mass spectrometry (for m6A) and direct RNA sequencing (for $5 \mathrm{mC}$ ) $[17,18,24]$. Our m6A pattern is based on sequencing data from two replicates. Although some positions are consistent between two experiments (e.g., 27,764), others vary (e.g., 28,616). The analysis of the output of m6Anet program shows that the positions putatively methylated have a significantly higher probability $(>25 \%)$ than the putatively non-methylated $(<5 \%)$, which is consistent with the model training and benchmarking [33]. Therefore, positions that, in at least one experiment, have a $\geq 50 \%$ probability are indicative that a substantial proportion of modified bases, over unmodified, are present in the sample. The m6A detection method employed here uses a model that takes into account the mixture of modified and unmodified RNAs and outputs the m6A-modification probability at any given site for all the DRACH 5-mers represented in the neural network training data and this might, at least in part, explain the variance among experiments as observed with different cell lines [33]. As a future perspective, the m6Anet model can be trained for the SARS-CoV-2 genome, which might adjust the probability values for m6A prediction in these specific RNAs.

As expected, the DRACH pattern is highly conserved among SARS-CoV-2 variants, although, in at least one position, a significant mutation was observed in the nucleocapsid region in variants Beta and Eta (Figure 2) that could negatively affect methylation by disrupting the DRACH motif [28]. The DRACH motif is highly conserved and strongly associated with m6A methylation (in the middle " $\mathrm{A}$ ") and, therefore, provides another level for selective pressure on SARS-CoV-2. The significance of this finding needs to be further investigated with comparative infection experiments using different combinations of viral and host cell lineages to determine the impact of DRACH mutations in viral growth. Additionally, differential methylation among the variants must be explored because experimental data with knockdown METTL3 viruses suggest that hypomethylated viral genomes are produced and the infection is significantly reduced [17].

As a future perspective, we are working on a methodology to allow full length, high coverage sequencing of the whole SARS-CoV-2 RNA genome by direct RNA sequencing and, therefore, extend the m6A analysis to ORF1ab, Spike and the $5^{\prime}$-untranslated region to compare the differential methylation among lineages and variants.

Author Contributions: Conceptualization, M.R.S.B., J.H.C.C. and L.M.R.J.; methodology, M.R.S.B., J.T.M., J.H.C.C. and C.T.B.; software, M.R.S.B., J.H.C.C. and F.A.; validation, J.H.C.C.; writing-original draft preparation, M.R.S.B.; writing - review and editing, all authors; funding acquisition, M.R.S.B., L.M.R.J. and C.T.B. All authors have read and agreed to the published version of the manuscript.

Funding: This work was supported by Fundação de Amparo à Pesquisa do Estado de São Paulo (FAPESP), Brazil, grant 2020/08943-5 to L.M.J., M.R.S.B. and F.A. and Conselho Nacional de Desenvolvimento Científico e Tecnológico (CNPq), Brazil, grant 405691/2018-1 to C.T.B. and grant 303912/2017-0 to M.R.S.B.

Data Availability Statement: The data presented in this study are available on request from the corresponding author. The data are not publicly available until the manuscript is accepted for publication.

Acknowledgments: The authors thank José Luiz Proença-Módena (University of Campinas-UNICAMP, SP, Brazil) for providing the SARS-CoV-2 sample and Fernanda Prieto, (Interprise, Inc.) for suggestions and assistance with Nanopore supply acquisitions.

Conflicts of Interest: The authors declare no conflict of interest.

\section{References}

1. Hogle, J.M. Poliovirus Cell Entry: Common Structural Themes in Viral Cell Entry Pathways. Annu. Rev. Microbiol. 2002, 56, 677-702. [CrossRef]

2. Guerra, F.M.; Bolotin, S.; Lim, G.; Heffernan, J.; Deeks, S.L.; Li, Y.; Crowcroft, N.S. The basic reproduction number (R0) of measles: A systematic review. Lancet Infect. Dis. 2017, 17, e420-e428. [CrossRef] 
3. Krammer, F.; Smith, G.J.D.; Fouchier, R.A.M.; Peiris, M.; Kedzierska, K.; Doherty, P.C.; Palese, P.; Shaw, M.L.; Treanor, J.; Webster, R.G.; et al. Influenza. Nat. Rev. Dis. Primer 2018, 4, 1-21. [CrossRef] [PubMed]

4. Islam, M.A.; Kundu, S.; Alam, S.S.; Hossan, T.; Kamal, M.A.; Hassan, R. Prevalence and characteristics of fever in adult and paediatric patients with coronavirus disease 2019 (COVID-19): A systematic review and meta-analysis of 17515 patients. PLoS ONE 2021, 16, e0249788. [CrossRef]

5. Wang, C.; Wang, Z.; Wang, G.; Lau, J.Y.-N.; Zhang, K.; Li, W. COVID-19 in early 2021: Current status and looking forward. Signal Transduct. Target. Ther. 2021, 6, 1-14. [CrossRef]

6. Baltimore, D. Expression of animal virus genomes. Bacteriol. Rev. 1971, 35, 235-241. [CrossRef] [PubMed]

7. Dill, J.A.; Camus, A.C.; Leary, J.H.; Di Giallonardo, F.; Holmes, E.C.; Ng, T.F.F. Distinct Viral Lineages from Fish and Amphibians Reveal the Complex Evolutionary History of Hepadnaviruses. J. Virol. 2016, 90, 7920-7933. [CrossRef] [PubMed]

8. Jiang, S.; Xia, S.; Ying, T.; Lu, L. A novel coronavirus (2019-nCoV) causing pneumonia-associated respiratory syndrome. Cell. Mol. Immunol. 2020, 17, 554. [CrossRef]

9. Hanff, T.C.; Mohareb, A.M.; Giri, J.; Cohen, J.B.; Chirinos, J.A. Thrombosis in COVID-19. Am. J. Hematol. 2020, 95, 1578-1589. [CrossRef]

10. Almeida, J.D.; Berry, D.M.; Cunningham, C.H.; Hamre, D.; Hofstad, M.S.; Mallucci, L.; McInstosh, D.A.; Tyrrell, D.A.J. Virology: Coronaviruses. Nature 1968, 220, 650. [CrossRef]

11. Cui, J.; Li, F.; Shi, Z.-L. Origin and evolution of pathogenic coronaviruses. Nat. Rev. Microbiol. 2019, 17, 181-192. [CrossRef] [PubMed]

12. Wu, C.; Liu, Y.; Yang, Y.; Zhang, P.; Zhong, W.; Wang, Y.; Wang, Q.; Xu, Y.; Li, M.; Li, X.; et al. Analysis of therapeutic targets for SARS-CoV-2 and discovery of potential drugs by computational methods. Acta Pharm. Sin. B 2020, 10, 766-788. [CrossRef] [PubMed]

13. V'kovski, P.; Kratzel, A.; Steiner, S.; Stalder, H.; Thiel, V. Coronavirus biology and replication: Implications for SARS-CoV-2. Nat. Rev. Microbiol. 2021, 19, 155-170. [CrossRef] [PubMed]

14. Brocard, M.; Ruggieri, A.; Locker, N. m6A RNA methylation, a new hallmark in virus-host interactions. J. Gen. Virol. 2017, 98, 2207-2214. [CrossRef]

15. Gokhale, N.S.; McIntyre, A.B.R.; McFadden, M.J.; Roder, A.E.; Kennedy, E.M.; Gandara, J.A.; Hopcraft, S.E.; Quicke, K.M.; Vazquez, C.; Willer, J.; et al. N6-Methyladenosine in Flaviviridae Viral RNA Genomes Regulates Infection. Cell Host Microbe 2016, 20, 654-665. [CrossRef] [PubMed]

16. Baquero-Perez, B.; Geers, D.; Díez, J. From A to m6A: The Emerging Viral Epitranscriptome. Viruses 2021, 13, 1049. [CrossRef] [PubMed]

17. Li, N.; Hui, H.; Bray, B.; Gonzalez, G.M.; Zeller, M.; Anderson, K.G.; Knight, R.; Smith, D.; Wang, Y.; Carlin, A.F.; et al. METTL3 regulates viral m6A RNA modification and host cell innate immune responses during SARS-CoV-2 infection. Cell Rep. 2021, 35, 109091. [CrossRef]

18. Liu, J.; Xu, Y.-P.; Li, K.; Ye, Q.; Zhou, H.-Y.; Sun, H.; Li, X.; Yu, L.; Deng, Y.-Q.; Li, R.-T.; et al. The m6A methylome of SARS-CoV-2 in host cells. Cell Res. 2021, 31, 404-414. [CrossRef]

19. Gatsiou, A.; Stellos, K. Dawn of Epitranscriptomic Medicine. Circ. Genomic Precis. Med. 2018, 11, e001927. [CrossRef] [PubMed]

20. Nazario-Toole, A.E.; Xia, H.; Gibbons, T.F. Whole-genome Sequencing of SARS-CoV-2: Using Phylogeny and Structural Modeling to Contextualize Local Viral Evolution. Mil. Med. 2021, usab031. [CrossRef] [PubMed]

21. Kietrys, A.M.; Velema, W.A.; Kool, E.T. Fingerprints of Modified RNA Bases from Deep Sequencing Profiles. J. Am. Chem. Soc. 2017, 139, 17074-17081. [CrossRef]

22. Jenjaroenpun, P.; Wongsurawat, T.; Wadley, T.D.; Wassenaar, T.M.; Liu, J.; Dai, Q.; Wanchai, V.; Akel, N.S.; Jamshidi-Parsian, A.; Franco, A.T.; et al. Decoding the epitranscriptional landscape from native RNA sequences. Nucleic Acids Res. 2021,49 , e7. [CrossRef] [PubMed]

23. Bull, R.A.; Adikari, T.N.; Ferguson, J.M.; Hammond, J.M.; Stevanovski, I.; Beukers, A.G.; Naing, Z.; Yeang, M.; Verich, A.; Gamaarachchi, H.; et al. Analytical validity of nanopore sequencing for rapid SARS-CoV-2 genome analysis. Nat. Commun. 2020, 11, 6272. [CrossRef] [PubMed]

24. Kim, D.; Lee, J.-Y.; Yang, J.-S.; Kim, J.W.; Kim, V.N.; Chang, H. The Architecture of SARS-CoV-2 Transcriptome. Cell 2020, 181, 914-921.e10. [CrossRef] [PubMed]

25. Miladi, M.; Fuchs, J.; Maier, W.; Weigang, S.; i Pedrosa, N.D.; Weiss, L.; Lother, A.; Nekrutenko, A.; Ruzsics, Z.; Panning, M.; et al. The Landscape of SARS-CoV-2 RNA Modifications. bioRxiv 2020. [CrossRef]

26. Taiaroa, G.; Rawlinson, D.; Featherstone, L.; Pitt, M.; Caly, L.; Druce, J.; Purcell, D.; Harty, L.; Tran, T.; Roberts, J.; et al. Direct RNA sequencing and early evolution of SARS-CoV-2. bioRxiv 2020. [CrossRef]

27. Vacca, D.; Fiannaca, A.; Tramuto, F.; Cancila, V.; Paglia, L.L.; Mazzucco, W.; Gulino, A.; Rosa, M.L.; Maida, C.M.; Morello, G.; et al. Direct RNA nanopore sequencing of SARS-CoV-2 extracted from critical material from swabs. bioRxiv 2020. [CrossRef]

28. Bayoumi, M.; Munir, M. Evolutionary conservation of the DRACH signatures of potential N6-methyladenosine (m6A) sites among influenza A viruses. Sci. Rep. 2021, 11, 4548. [CrossRef]

29. Araujo, D.B.; Machado, R.R.G.; Amgarten, D.E.; Malta, F.d.M.; de Araujo, G.G.; Monteiro, C.O.; Candido, E.D.; Soares, C.P.; de Menezes, F.G.; Pires, A.C.C.; et al. SARS-CoV-2 isolation from the first reported patients in Brazil and establishment of a coordinated task network. Mem. Inst. Oswaldo Cruz 2020, 115, e200342. [CrossRef] [PubMed] 
30. Hoffmann, M.; Kleine-weber, H.; Schroeder, S.; Krüger, N.; Herrler, T.; Erichsen, S.; Schiergens, T.S.; Herrler, G.; Wu, N.-H.; Nitsche, A.; et al. SARS-CoV-2 Cell Entry Depends on ACE2 and TMPRSS2 and Is Blocked by a Clinically Proven Protease Inhibitor. Cell 2020, 181, 271-280. [CrossRef]

31. Li, H. Minimap2: Pairwise alignment for nucleotide sequences. Bioinforma. Oxf. Engl. 2018, 34, 3094-3100. [CrossRef] [PubMed]

32. Li, H.; Handsaker, B.; Wysoker, A.; Fennell, T.; Ruan, J.; Homer, N.; Marth, G.; Abecasis, G.; Durbin, R.; 1000 Genome Project Data Processing Subgroup. The Sequence Alignment/Map format and SAMtools. Bioinformatics 2009, 25, 2078-2079. [CrossRef] [PubMed]

33. Hendra, C.; Pratanwanich, P.N.; Wan, Y.K.; Goh, W.S.S.; Thiery, A.; Göke, J. Detection of m6A from direct RNA sequencing using a Multiple Instance Learning framework. bioRxiv 2021. [CrossRef]

34. Hendra, C.; Wan, Y.K. GoekeLab/m6anet: Pre-Release; Zenodo: Genève, Switzerland, 2021.

35. Chen, Y.; Davidson, N.M.; Wan, Y.K.; Patel, H.; Yao, F.; Low, H.M.; Hendra, C.; Watten, L.; Sim, A.; Sawyer, C.; et al. A systematic benchmark of Nanopore long read RNA sequencing for transcript level analysis in human cell lines. bioRxiv 2021. [CrossRef]

36. Meng, Y.; Zhang, Q.; Wang, K.; Zhang, X.; Yang, R.; Bi, K.; Chen, W.; Diao, H. RBM15-mediated N6-methyladenosine modification affects COVID-19 severity by regulating the expression of multitarget genes. Cell Death Dis. 2021, 12, 1-10. [CrossRef]

37. Ahmed-Belkacem, R.; Sutto-Ortiz, P.; Guiraud, M.; Canard, B.; Vasseur, J.-J.; Decroly, E.; Debart, F. Synthesis of adenine dinucleosides SAM analogs as specific inhibitors of SARS-CoV nsp14 RNA cap guanine-N7-methyltransferase. Eur. J. Med. Chem. 2020, 201, 112557. [CrossRef]

38. Paramasivam, A. RNA 2'-O-methylation modification and its implication in COVID-19 immunity. Cell Death Discov. 2020, 6, 118. [CrossRef] 